Apuntes Universitarios, 2022: 12 (1), enero-marzo

ISSN: 2304-0335 DOI: https://doi.org/10.17162/au.v11i5.922

\title{
Programa basado en técnicas artísticas para incrementar la inteligencia emocional en jóvenes voluntarios
}

\author{
Artistic techniques-based program to increase emotional intelligence \\ in young volunteers \\ Claudia Chirinos-Lizarraga, ${ }^{1}$ Grecia Reyes-Tejada, ${ }^{2}$ Ventura-León, José ${ }^{3}$, \\ Mario Reyes-Bossio ${ }^{4 a}$
}

Facultad de Psicología, Universidad Peruana de Ciencias Aplicadas (UPC), Lima, Perú ${ }^{1,2,4}$ Universidad Privada del Norte, Lima, Perú (UPN) ${ }^{3}$

ORCID ID: http://orcid.org/0000-0003-5655-1927 1

D ORCID ID: http://orcid.org/0000-0002-1450-474X ${ }^{2}$

(iD) ORCID ID: http://orcid.org/0000-0003-2996-4244 3

iD ORCID ID: http://orcid.org/0000-0003-2641-92834

Recibido: 25 de abril de 2021

Aceptado: 09 de setiembre de 2021

\section{Resumen}

Este estudio tuvo como propósito identificar los efectos del programa ART-EMO en los niveles de inteligencia emocional de jóvenes voluntarios que trabajan con niños en una entidad privada. La muestra fue compuesta por 10 participantes cuyas edades oscilaban los 21 y 30 años; el 10\% eran hombres y el $90 \%$ mujeres. Se aplicó, en dos condiciones de tiempo, el Inventario de Inteligencia Emocional en adultos: EQ-i-M20 creado por Pérez-Fuentes et al. (2014) y validado en una población de estudiantes peruanos por Domínguez-Lara et al. (2017). Para fines de la presente investigación, en el 2017 se elaboró el programa ART-EMO mediante un diseño cuasi experimental con un solo grupo, considerándose a Guerrón (2015) como referente. El programa tuvo un impacto alto a nivel total de la inteligencia emocional $(d=.82)$ y en sus dimensiones intrapersonal $(d=1.04)$, interpersonal $(d=.90)$ y adaptabilidad $(d=1.01)$; no obstante, en estado de ánimo $(d=.67)$ y manejo de estrés $(d=.55)$, fue moderado. A modo de conclusión, el programa fue eficaz y con ello se busca que las instituciones fomenten el desarrollo emocional en quienes son voluntarios.

Palabras claves: Inteligencia emocional, educación, programa ART-EMO, jóvenes, voluntarios

\begin{abstract}
The purpose of this study was to identify the effect of the ART-EMO program on the levels of emotional intelligence of young volunteers working with children in a private entity. The sample
\end{abstract}

${ }^{a}$ Correspondencia al autor: 
consisted of 10 participants whose ages ranged from 21 to 30 years; $10 \%$ were men and $90 \%$ were women. Inventory of Emotional Intelligence in Adults: EQ-i-M20 created by Pérez-Fuentes et al. (2014) and validated in Peruvian students by Domínguez-Lara et al. (2017) was applied in twotimes conditions. For research purposes, in 2017, the ART-EMO program was created using a quasi-experimental design with a single group, taking as reference Guerrón (2015). The program had a high impact at the total level of Emotional Intelligence $(\mathrm{d}=.82)$ and in its Intrapersonal $(\mathrm{d}=$ 1.04), Interpersonal $(\mathrm{d}=.90)$ and Adaptability $(\mathrm{d}=1.01)$ dimensions; however, in General Mood $(\mathrm{d}=.67)$ and Stress Management $(\mathrm{d}=.55)$, it was moderate. In conclusion, the program was effective; and it is sought that institutions promote emotional development in volunteers.

Keywords: Emotional intelligence, education, ART-EMO program, young, volunteers

\section{Introducción}

La interacción entre seres humanos facilita el desarrollo de habilidades sociales y la adaptación con el entorno. A su vez, brinda beneficios a nivel personal y grupal (Betina \& Norma, 2017; Mesa, 2018). Ante ello, el servicio de voluntariado es una interacción entre personas que realizan actividades de interés sin retribución económica, incentivados por su deseo de ayudar a otros y transformar la sociedad (Huerta, 2017; Ros-Morente et al., 2018). El último reporte del programa de Voluntariados de las Naciones Unidas (VNU) del 2018 indicó que, un billón de personas prestó ayuda no remunerada. En Perú, hasta el 2017, la tasa de voluntariado era de 16\%, de la fuerza laboral con un mínimo de 14 años de edad (International Labour Organization [ILOSTAT], 2020).

El voluntario atraviesa constantes cambios, y adquiere conocimientos y habilidades en la etapa de la juventud (Mesa, 2018; Quiroga \& Chamblás, 2014). Sin embargo, tiende a responder con impulsividad y/o agresividad, tanto consigo mismo como con los demás (Quintero et al., 2020). Por ende, el voluntario que trabaja con niños debe contar con comunicación asertiva, empatía, liderazgo y proactividad, además de manejar su frustración (Huerta, 2017; Zambrano et al., 2017).

En este contexto, el desarrollo de la inteligencia emocional (IE) potencia las habilidades mencionadas, definiéndose como el conjunto de competencias y habilidades personales, emocionales y sociales relacionadas entre sí, que permiten un adecuado desenvolvimiento en la sociedad (BarOn, 2006). La IE abarca cinco dimensiones: intrapersonal (entender y expresar emociones), interpersonal (comprender emociones de otros y relacionarse adecuadamente), manejo de estrés (modo de reaccionar ante adversidades), adaptabilidad (dar soluciones efectivas en situaciones de cambio) y estado de ánimo general (disfrutar de actividades), según BarOn (2006). 
De esta manera, su desarrollo favorece la estabilidad emocional y fomenta la creatividad (PérezFuentes et al., 2014).

Respecto a lo anterior, existen intervenciones para el desarrollo de la IE en diferentes poblaciones, tales como niños (Coz et al., 2020), adolescentes (Sigüenza-Marín, Fonseca-Pedrero \& Perez-Albeniz, 2019) y adultos mayores (Pérez-Fuentes et al., 2014) con óptimos resultados, así como programas basados en la teoría de BarOn, (Gaspar, 2015; Huemura, 2018; Mamani-Benito et al., 2018). Para mayor detalle véase Tabla 1.

\section{Tabla 1}

Programas de inteligencia emocional

\begin{tabular}{|c|c|c|c|}
\hline Secciones & PRM & $\begin{array}{l}\text { Educación Emocional para } \\
\text { adolescentes }\end{array}$ & $\begin{array}{c}\text { Programa para desarrollar la } \\
\text { inteligencia emocional }\end{array}$ \\
\hline Autores & Gaspar (2015) & Huemura (2018) & $\begin{array}{c}\text { Mamani-Benito, Brousett-Minaya, } \\
\text { Ccori-Zúñiga, y Villasante-Idme (2017) }\end{array}$ \\
\hline Participantes & $\begin{array}{l}24 \text { universitarios de la } \\
\text { carrera de Ingeniería }\end{array}$ & $\begin{array}{c}40 \text { estudiantes entre } 11 \text { y } 12 \\
\text { años }\end{array}$ & $\begin{array}{c}33 \text { adolescentes mujeres de quinto de } \\
\text { secundaria }\end{array}$ \\
\hline $\begin{array}{l}\text { Número de } \\
\text { sesiones }\end{array}$ & 14 & 12 & 10 \\
\hline $\begin{array}{l}\text { Duración de } \\
\text { cada sesión }\end{array}$ & 2 horas & 2 horas & 45 minutos \\
\hline $\begin{array}{l}\text { Duración de } \\
\text { programa }\end{array}$ & 14 semanas & 10 semanas & 5 meses \\
\hline Teoría de IE & BarOn (2000) & BarOn (2000) & BarOn (2000) \\
\hline $\begin{array}{l}\text { Técnicas } \\
\text { utilizadas }\end{array}$ & $\begin{array}{l}\text { Material tecnológico } \\
\text { (computadoras) }\end{array}$ & & $\begin{array}{l}\text { Dinámicas de integración, } \\
\text { dramatización y reflexión }\end{array}$ \\
\hline
\end{tabular}

Por otro lado, los programas de intervención de IE en universitarios demostraron eficacia al incrementarse las puntuaciones en tres de sus componentes, mas no en el manejo del estrés y estado de ánimo general (Gaspar, 2015). En su aplicación con adolescentes, se evidenció mayor puntaje en las dimensiones "estado de ánimo general” y "adaptabilidad" (Huemura, 2018); mientras que otro programa en la misma población reveló un impacto grande en la dimensión “interpersonal” (Mamani-Benito et al., 2018). En general, las intervenciones en IE han demostrado eficacia para identificar emociones básicas, alcanzar mayor autoconocimiento, adquirir alternativas de afrontamiento y aprender a controlar impulsos (Coz et al., 2020).

Asimismo, el desarrollo de la IE se logra con mayor efectividad al utilizar técnicas artísticas (Malchiodi, 2010), consideradas como herramientas de expresión emocional y crecimiento personal (Mayo, 2018), basadas en la arteterapia y dirigidas a distintas personas (Klein, 2006). A través de creaciones sonoras, manuales, gráficas y escritas, los jóvenes desarrollan otra forma de 
razonar sus conflictos personales para reestablecer su equilibrio emocional (Guerrón, 2015; Klein, 2006; Mayo, 2018). Las evidencias empíricas demuestran la eficacia de su uso en personas de diferentes edades con dificultades emocionales; no obstante, se cuenta con poca evidencia en voluntarios (Castillo, 2019; Coz et al., 2020; Rojas \& Galván, 2020; Guerrón, 2015). Estos se explican con detalle en la Tabla 2.

\section{Tabla 2}

Programas de Técnicas artísticas

\begin{tabular}{|c|c|c|c|}
\hline Secciones & $\begin{array}{l}\text { Guía didáctica en Arteterapia } \\
\text { para disminuir la ansiedad }\end{array}$ & $\begin{array}{l}\text { "Meditarte" Diseño de un } \\
\text { programa de Arteterapia y } \\
\text { Mindfulness para mujeres con } \\
\text { dolor crónico en atención } \\
\text { primaria }\end{array}$ & $\begin{array}{l}\text { Arteterapia: una experiencia } \\
\text { de implementación remedial } \\
\text { voluntaria en universitarios }\end{array}$ \\
\hline Autores & Guerrón (2015) & Castillo (2019) & Rojas y Galván (2020) \\
\hline Participantes & $\begin{array}{l}10 \text { estudiantes de quinto de } \\
\text { secundaria y primero de } \\
\text { Bachillerato }\end{array}$ & $\begin{array}{l}8 \text { mujeres autovalentes en edades } \\
\text { comprendidas entre } 45 \text { y } 70 \text { años }\end{array}$ & $\begin{array}{c}10 \text { jóvenes universitarios ( } 3 \\
\text { hombres y } 7 \text { mujeres) entre } 19 \\
\text { y } 22 \text { años de edad }\end{array}$ \\
\hline $\begin{array}{l}\text { Número de } \\
\text { sesiones }\end{array}$ & 12 & 13 & 10 \\
\hline $\begin{array}{l}\text { Duración de } \\
\text { cada sesión }\end{array}$ & 2 horas & 2 horas y 30 minutos & 2 horas \\
\hline $\begin{array}{c}\text { Duración de } \\
\text { programa }\end{array}$ & 6 semanas & 3 meses y medio & 1 semestre académico \\
\hline $\begin{array}{l}\text { Técnicas } \\
\text { utilizadas }\end{array}$ & $\begin{array}{l}\text { Collage, máscaras, mapa de } \\
\text { emociones, autorretrato, mi } \\
\text { "yo" fuerte y mi "yo" débil, "Lo } \\
\text { prohibido", mandala, } \\
\text { dramaterapia, "Animal que me } \\
\text { represente", diario, "Mi objeto } \\
\text { favorito" y "Exprésate" }\end{array}$ & $\begin{array}{l}\text { Autoreporte con medición de } \\
\text { EVA de inicio y final, autoreporte } \\
\text { con reflexión individual sobre las } \\
\text { creaciones narrativas, de escritura } \\
\text { y pintura }\end{array}$ & $\begin{array}{l}\text { Danzaterapia y actividades de } \\
\text { trabajo corporal plástico }\end{array}$ \\
\hline
\end{tabular}

De este modo, la guía didáctica de Guerrón (2015) muestra disminución de la ansiedad en jóvenes e incremento de la escucha activa. Situación similar se presenta en adultas, donde tuvo un impacto positivo en las consecuencias emocionales a causa del dolor físico (Castillo, 2019); mientras que en niños promueve cambios en su conducta y fomenta actitudes positivas (Coz et al., 2020) y en universitarios revela un incremento en sus habilidades interpersonales (Rojas \& Galván, 2020). En ese sentido, esta intervención se justifica en tres aspectos: (a) teórico, porque amplificará el marco conceptual de la IE en voluntarios; (b) práctico, debido a que la implementación con técnicas artísticas permitirá conocer los efectos del mismo para aplicarlo en otros contextos; (c) 
social, ya que el impacto del programa recaerá en la labor de los voluntarios, lo que favorecerá a la organización y población beneficiaria (Poyatos, 2013; Quiroga \& Chamblás, 2014).

En base a lo mencionado, esta investigación tiene como objetivo general determinar los efectos del programa ART-EMO en la IE en un grupo de voluntarios de Lima. Los objetivos específicos incluyen la identificación las puntuaciones de las dimensiones de la variable antes del programa, así como su comparación antes y después de la intervención. Todo ello mediante un diseño cuasi-experimental con un solo grupo (Alarcón, 2008; Ato et al., 2013; Reyes-Bossio, Raimundi \& Gómez, 2012; Trujillo-Torrealva \& Reyes-Bossio, 2019).

\section{Método}

\section{Participantes}

La muestra estuvo conformada por 10 jóvenes voluntarios que dictan clases a niños en una organización privada de Lima, Perú, quienes aceptaron participar de manera voluntaria del programa de intervención. El 90\% ( $n=9)$ fueron mujeres y el 10\% $(n=1)$ hombres, con edades comprendidas entre los 21 y 30 años $\left(\mu=24.9, \sigma^{2}=3.07\right)$. El 20\% $(n=2)$ estudiaba administración y el $80 \%(n=8)$ psicología; el 100\% $(n=10)$ consideraba la relación con sus compañeros como buena; y el 100\% $(n=10)$ deseaba desarrollar habilidades sociales y emocionales. Se consideró a jóvenes que realicen actividades con niños por lo menos una vez a la semana en un voluntariado. Con el fin de evaluar el efecto del programa, el grupo experimental estuvo conformado por uno solo, al cual se le administró el instrumento de IE a todos los participantes $(n=10)$.

Para establecer el número de participantes se tomó como referencia los programas de Reyes-Bossio, Raimundi y Gómez, (2012); Guerrón (2015), Rojas y Galvan (2020); TrujilloTorrealva y Reyes-Bossio (2019), cuyas muestras fueron entre 10 y 16 participantes; y el programa de Jiménez et al. (2016) que contó con nueve participantes. Asimismo, Sánchez-Gómez et al. (2021) plantean que un programa de intervención es efectivo al contar con un promedio de 13 participantes, puesto que se fomenta la cohesión entre pares.

\section{Instrumentos}

\section{Inventario de inteligencia emocional}

$E Q-i-M 20$, creado por Pérez-Fuentes et al. (2014), cuya adaptación peruana fue realizada por Domínguez-Lara et al. (2017). Cuenta con evidencias de validez basadas en el contenido por jueces 
expertos, quienes recomendaron reemplazar la palabra "furia" por "cólera" del ítem 2 ("Me resulta difícil controlar mi ira [cólera]"); de igual manera la palabra "enfadar" por "enojar" en el ítem 12 (“Me enfado [enojo] con facilidad”) (Domínguez-Lara et al., 2017). Respecto a la fiabilidad, se obtuvieron índices aceptables para las cinco dimensiones, encontrándose Intrapersonal $(\alpha=.81)$, Interpersonal $(\alpha=.65)$, Manejo de estrés $(\alpha=.82)$, Adaptabilidad $(\alpha=.72)$ y Estado de ánimo general $(\alpha=.88)$.

El inventario está constituido por 20 reactivos basados en el modelo de BarOn I-Ce, como se citó en Pérez-Fuentes et al. (2014), los cuales están compuestas por cinco dimensiones: Intrapersonal, Interpersonal, Manejo de estrés, Adaptabilidad y Estado de Ánimo General. Cada dimensión consta de cuatro reactivos respectivamente. El formato de respuesta es tipo Likert de cuatro puntos, que oscila desde uno ("nunca me pasa") a cuatro (“siempre me pasa").

\section{Programa de Intervención “ART-EMO”}

El programa de intervención ART-EMO (Emotions and Art) presentó como objetivo incrementar la inteligencia emocional de jóvenes voluntarios a través del empleo de técnicas artísticas. Se tomó como referencia la Guía didáctica en Arteterapia para disminuir la Ansiedad de Guerrón (2015), el programa "Meditarte" de Castillo (2019) y Programa de Arteterapia de Rojas y Galván (2020) para la elaboración de este programa.

El programa se desarrolló durante dos semanas, y estuvo compuesto por cinco módulos, constituidos por 10 sesiones grupales (dos por cada módulo) de dos horas de duración cada una, con una frecuencia interdiaria. Al realizar dos sesiones por fecha, hubo un receso de 30 minutos entre ambas, con un total de cuatro horas y media cada módulo. Asimismo, se incorporó una sesión de presentación en la que se aplicó el pretest, y otra sesión de finalización, en el que se efectuó el postest.

Para establecer el número de sesiones, se tomó como referencia el programa realizado por Mamani-Benito et al. (2018) (véase Tabla 1). Otras investigaciones realizadas para desarrollar la IE han elaborado programas con un número de sesiones entre ocho y doce, con un rango de duración entre 90 y 180 minutos correspondientemente, lográndose un incremento de la variable ya mencionada (Murrieta et al., 2014; Ramos et al., 2009; Trujillo-Torrealva \& Reyes-Bossio, 2019). Asimismo, para establecer la frecuencia de las sesiones se tomó como referencia los programas de Guerrón (2015), y Trujillo-Torrealva y Reyes-Bossio (2019), cuya frecuencia de sesiones fue de dos veces por semana en ambos casos. 
Las herramientas empleadas en este programa fueron técnicas artísticas, donde se incluían dinámicas de conexión y la experiencia de arte como tal. Además, se incluyó un momento psicoeducativo en el que se elaboró una explicación grupal sobre el tema aplicado después de atravesar la sesión (ver Tabla 3). Es importante mencionar que este programa pasó por una evaluación de contenido por cinco expertos con maestría en desarrollo de proyectos y programas sociales, especialistas en diseño de talleres psicoeducativos, así como especialistas en técnicas artísticas aplicadas a la psicología. La evaluación de los jueces indicó que cada sesión debería tener un nombre y un tema, verificar que cada actividad corresponda al objetivo planteado y delimitar cuidadosamente el trabajo introspectivo y el colectivo en los módulos Intra e Interpersonal. A su vez, la misma aplicación del programa y los resultados obtenidos representa otra forma de validarlo.

\section{Tabla 3}

Programa Art-Emo

\begin{tabular}{|c|c|c|c|c|c|c|}
\hline \multirow[b]{2}{*}{ Módulo } & \multirow[b]{2}{*}{$\begin{array}{c}\mathrm{N}^{\circ} \\
\text { Sesión }\end{array}$} & \multirow[b]{2}{*}{ Tema } & \multirow[b]{2}{*}{ Objetivos } & \multicolumn{2}{|c|}{ Técnicas artísticas } & \multirow[b]{2}{*}{ Tiempo } \\
\hline & & & & $\begin{array}{l}\text { Dinámicas de } \\
\text { conexión }\end{array}$ & $\begin{array}{c}\text { Experiencia de } \\
\text { arte }\end{array}$ & \\
\hline \multirow[t]{2}{*}{ Intrapersonal } & 1 & Asertividad & $\begin{array}{c}\text { Potencializar la habilidad para comprender, } \\
\text { aceptar y expresar sentimientos propios, sin } \\
\text { afectar a otro. }\end{array}$ & $\begin{array}{l}\text { Dinámica: } \\
\text { "Colimage" }\end{array}$ & “Tú eres" & $\begin{array}{l}120 \\
\text { minutos }\end{array}$ \\
\hline & 2 & Autonomía & $\begin{array}{l}\text { Potencializar la seguridad en la toma de } \\
\text { decisiones, y disfrutar de las actividades. }\end{array}$ & $\begin{array}{l}\text { Dinámica: "El } \\
\text { ciego de la pareja" }\end{array}$ & "Máscaras" & $\begin{array}{l}120 \\
\text { minutos }\end{array}$ \\
\hline \multirow[t]{2}{*}{ Interpersonal } & 3 & $\begin{array}{l}\text { Relaciones } \\
\text { interpersonal } \\
\text { es positivas }\end{array}$ & $\begin{array}{l}\text { Potencializar la habilidad para identificar, } \\
\text { comprender y apreciar los sentimientos de } \\
\text { los demás. }\end{array}$ & $\begin{array}{c}\text { Dinámica: } \\
\text { "Garabateando" }\end{array}$ & "CreaPoema" & $\begin{array}{l}120 \\
\text { minutos }\end{array}$ \\
\hline & 4 & $\begin{array}{l}\text { Participación } \\
\text { activa }\end{array}$ & $\begin{array}{l}\text { Potencializar el sentido de contribución y } \\
\text { cooperación en grupos sociales. }\end{array}$ & $\begin{array}{l}\text { Dinámica: "Árbol, } \\
\text { manzana, gusano" }\end{array}$ & $\begin{array}{l}\text { "Creando } \\
\text { juntos" }\end{array}$ & $\begin{array}{c}120 \\
\text { minutos }\end{array}$ \\
\hline \multirow[b]{2}{*}{ Adaptabilidad } & 5 & $\begin{array}{l}\text { Resolución } \\
\text { de conflictos }\end{array}$ & $\begin{array}{l}\text { Potencializar la habilidad para identificar } \\
\text { problemas y dar soluciones efectivas. }\end{array}$ & $\begin{array}{l}\text { Dinámica: } \\
\text { "Superlución", }\end{array}$ & "RetAndo" & $\begin{array}{l}120 \\
\text { minutos }\end{array}$ \\
\hline & 6 & Flexibilidad & $\begin{array}{l}\text { Potencializar la capacidad de adaptación a } \\
\text { situaciones de cambio. }\end{array}$ & $\begin{array}{l}\text { Dinámica: } \\
\text { "Expresándome a } \\
\text { través del } \\
\text { movimiento" }\end{array}$ & $\begin{array}{l}\text { "Obra de teatro } \\
\text { con guión } \\
\text { desconocido" }\end{array}$ & $\begin{array}{l}120 \\
\text { minutos }\end{array}$ \\
\hline \multirow{2}{*}{$\begin{array}{l}\text { Manejo de } \\
\text { estrés }\end{array}$} & 7 & $\begin{array}{l}\text { Manejo de } \\
\text { estrés }\end{array}$ & $\begin{array}{c}\text { Potencializar la habilidad de enfrentar activa } \\
\text { y positivamente el estrés ante situaciones } \\
\text { adversas. }\end{array}$ & $\begin{array}{l}\text { Dinámica: } \\
\text { "Nudo humano", }\end{array}$ & $\begin{array}{l}\text { "Una receta } \\
\text { loca" }\end{array}$ & $\begin{array}{l}120 \\
\text { minutos }\end{array}$ \\
\hline & 8 & $\begin{array}{l}\text { Control de } \\
\text { impulsos }\end{array}$ & $\begin{array}{l}\text { Potencializar la habilidad para manejar } \\
\text { emociones e impulsos y responder } \\
\text { asertivamente a situaciones adversas. }\end{array}$ & $\begin{array}{l}\text { Dinámica: } \\
\text { "No al visto de la } \\
\text { indiferencia" }\end{array}$ & "Mandala" & $\begin{array}{l}120 \\
\text { minutos }\end{array}$ \\
\hline \multirow{2}{*}{$\begin{array}{l}\text { Estado de } \\
\text { Ánimo General }\end{array}$} & 9 & $\begin{array}{l}\text { Actitud } \\
\text { positiva }\end{array}$ & $\begin{array}{l}\text { Potencializar la habilidad de mantener una } \\
\text { actitud positiva ante la adversidad. }\end{array}$ & $\begin{array}{l}\text { Dinámica } \\
\text { "La cruz y el } \\
\text { círculo" }\end{array}$ & $\begin{array}{l}\text { "Cerrando } \\
\text { conexiones" }\end{array}$ & $\begin{array}{l}120 \\
\text { minutos }\end{array}$ \\
\hline & 10 & Optimismo & $\begin{array}{c}\text { Potencializar la habilidad de mantener } \\
\text { sentimientos positivos y realizar actividades } \\
\text { gratificantes a fin de disfrutar la vida. }\end{array}$ & $\begin{array}{l}\text { Dinámica: } \\
\text { "DíaCrea" }\end{array}$ & $\begin{array}{l}\text { "Collage de } \\
\text { despedida" }\end{array}$ & $\begin{array}{l}120 \\
\text { minutos }\end{array}$ \\
\hline
\end{tabular}

\section{Procedimiento}


Los participantes fueron convocados tras una coordinación entre las investigadoras y las autoridades de una organización privada, luego de la aprobación de los lineamientos éticos para el presente estudio. Los voluntarios fueron evaluados de manera colectiva en medidas de pretest y postest en ambos momentos. Previo a esto, se obtuvo el consentimiento informado, el cual fue completado por los participantes para garantizar la confidencialidad del caso. Ambas evaluaciones se llevaron a cabo en las instalaciones de la organización, en un distrito de Lima en el mes de julio del 2018, en un día que no interfería con sus actividades de voluntariado. Los participantes acudieron a la evaluación y mostraron disposición para realizarla. En tal ocasión se les informó el objetivo del programa, además de exponer brevemente cuál sería la secuencia del mismo.

La intervención estuvo a cargo de las investigadoras y fue realizada en un espacio de la institución, con los materiales necesarios y con adecuada iluminación y ventilación. Los participantes asistieron durante dos semanas, de forma voluntaria, a la totalidad del programa ARTEMO, en el que desarrollaron actividades individuales y grupales basadas en técnicas artísticas durante dos horas cada sesión. Finalizada una sesión, se daba 30 minutos de receso y luego se continuaba con la siguiente. Se tomó en cuenta la asistencia para confirmar la cantidad de participantes que acudieron por sesión con la finalidad de registrarlo en una base de datos para los posteriores análisis. El programa tuvo una metodología individual y colectiva considerándose el modelo elaborado por otros autores (Castillo, 2019; Rojas y Galván, 2020; Guerrón, 2015).

Al inicio del programa, se generó rapport con los participantes a fin de establecer el encuadre y recoger sus expectativas. En cuanto al desarrollo de las sesiones, se comenzaba con una dinámica de conexión individual o grupal acorde al objetivo de cada módulo. En un segundo momento, realizaban actividades con técnicas artísticas, de forma individual o colectiva. Todas las actividades finalizaban con un feedback grupal. A modo de cierre y de psicoeducación, se les entregaba una ficha informativa sobre importancia de la IE sobre la labor que ejercen como voluntarios y las recomendaciones para mejorar su trabajo. La secuencia y especificaciones del programa se respetaron.

\section{Análisis estadísticos de los datos}

Se verificó la normalidad de cada dimensión mediante la prueba Shapiro-Wilk $(n<50)$, con la cual se determinó la prueba paramétrica $T$ de Student para muestras relacionadas en la comparación de las medias de cada dimensión y a nivel general entre el pre y el postest. Además, se obtuvo el 
tamaño del efecto mediante la $d$ de Cohen, junto al porcentaje de probabilidad de superioridad (PS) y el número necesario para tratar (NNT) basado en Ventura-León (2018). Los datos fueron analizados con el paquete estadístico SPSS versión 22.0 y Rstudio.

\section{Resultados}

En la Tabla 4 se muestran los estadísticos descriptivos obtenidos en la evaluación pretest y postest de la variable IE. En la primera, los evaluados presentaron puntuaciones promedio altas en forma general $(\mu=2.69)$ y en las dimensiones Intrapersonal $(\mu=2.75)$, Interpersonal $(\mu=3.13)$, Adaptabilidad $(\mu=2.93)$, Estado de ánimo general $(\mu=2.98)$ y Manejo de estrés $(\mu=3.05)$, pues los promedios obtenidos en cada una se acercaron al puntaje máximo posible de la escala Likert (va del 1 al 4). En la evaluación final, se evidencian los efectos de las técnicas artísticas en el aumento del puntaje promedio de las dimensiones en forma general $(\mu=3.31)$ y en las dimensiones Intrapersonal $(\mu=3.28)$, Interpersonal $(\mu=3.60)$, Adaptabilidad $(\mu=3.50)$, Estado de ánimo general $(\mu=3.35)$ y Manejo de estrés $(\mu=3.33)$; es decir, posiblemente la mayoría tenía conductas de IE con cierta frecuencia, y al final del programa esa frecuencia aumentó un poco más.

En base al análisis comparativo, se utilizó la prueba $T$ de Student para muestras relacionadas. Los resultados indican que si bien el programa no tuvo incremento estadísticamente significativo en la IE ( $t=1.87 ; p>.05)$ respecto a la significancia práctica (Cohen, 1988; VenturaLeón, 2018), obtuvo un tamaño del efecto grande ( $d=.8$ ) que, según la medida de Probabilidad de Superioridad $(P S)$, sugiere que existe un $72 \%$ de probabilidad de que al elegir al azar el puntaje de IE de un participante del postest y compararlo con uno del pretest, este sea mayor $(P S=.72)$; e incluso si se aumentase tres participantes en la muestra el impacto incrementaría (NNT=3.43) (Ventura-León, 2018). En las dimensiones, Intrapersonal ( $t=4.36, p<.05)$, Interpersonal $(t=3.28$, $p<.05)$, Adaptabilidad ( $t=3.04 ; p<.05)$, se obtuvo un incremento estadísticamente significativo con un tamaño del efecto grande $(d=1.04, d=.90, \mathrm{~d}=1.01)$; mientras que el Estado de ánimo general $(t=4.39 ; p<.05)$ presentó un tamaño de efecto moderado $(d=.62)$. No obstante, si bien en la dimensión Manejo de estrés, el efecto no fue estadísticamente significativo $(t=1.30 ; p>.05)$, el impacto fue moderado ( $d=.55)$. Además, según la PS, en todas las dimensiones evidencia que entre un $65 \%$ y $76 \%$ de probabilidad al elegir al azar el puntaje de un participante del postest y compararlo con uno del pretest, será superior; y según el NNT, si se aumentase entre tres a cinco participantes en la muestra el impacto incrementaría (ver Tabla 4). 
Nota: $\mu$ : media aritmética, $\sigma^{2}$ : desviación estándar, $p$ : significancia, $d$ : d de cohen (tamaño de efecto), PS (Probabilidad de Superioridad), NNT (Número Necesario para Tratar)

\section{Discusión}

El propósito de este estudio fue determinar los efectos del programa ART-EMO en la IE en un grupo de voluntarios de Lima. Los resultados sugieren que el programa incrementó las puntuaciones de la IE al obtenerse un tamaño del efecto considerado grande $(d>.80)$ (VenturaLeón, 2018). Este hallazgo indica que las personas incrementaron sus habilidades para entender y expresar cómo se sienten, su capacidad para comprender las emociones de los demás y relacionarse de forma adecuada, su modo de reaccionar ante las adversidades del entorno y su capacidad para dar soluciones efectivas a situaciones cambiantes, y disfrutar en todo momento de las actividades que realizan (BarOn, 2006). Se considera que el impacto del programa en esta muestra podría deberse a que los voluntarios se han visto interesados en trabajar estos aspectos de la IE; lo cual ha Tabla 4

Efectos del programa Art-Emo entre el pretest y el postest

\begin{tabular}{|c|c|c|c|c|c|c|c|c|c|}
\hline \multirow{2}{*}{ Dimensión } & \multicolumn{2}{|c|}{ Pretest } & \multicolumn{2}{|c|}{ Postest } & \multirow{2}{*}{$T$} & \multirow{2}{*}{$p$} & \multirow{2}{*}{$d$} & \multirow{2}{*}{$P S$} & \multirow{2}{*}{ NNT } \\
\hline & $\mu$ & $\sigma^{2}$ & $\mu$ & $\sigma^{2}$ & & & & & \\
\hline Intrapersonal & 2.75 & .54 & 3.28 & .48 & 4.36 & .002 & 1.04 & .76 & 2.74 \\
\hline Interpersonal & 3.13 & .55 & 3.60 & .49 & 3.28 & .010 & .90 & .73 & 3.09 \\
\hline Estado de Ánimo & 2.98 & .65 & 3.35 & .53 & 4.39 & .002 & .62 & .67 & 4.71 \\
\hline Adaptabilidad & 2.93 & .52 & 3.50 & .61 & 3.04 & .014 & 1.01 & .76 & 2.72 \\
\hline Manejo de Estrés & 3.05 & .55 & 3.33 & .46 & 1.30 & .227 & .55 & .65 & 5.40 \\
\hline Inteligencia Emocional & 2.69 & .26 & 3.31 & 1.04 & -1.87 & .095 & .82 & .72 & 3.43 \\
\hline
\end{tabular}

servido como herramienta para que exploren en sí mismos, reflexionen sobre sus acciones y lo lleven a su quehacer, pues desean brindar algo de ellos a los niños. Estos hallazgos son similares a los obtenidos por Gaspar (2015) y Mamani-Benito et al. (2018) donde los programas de intervención tuvieron un efecto grande en la inteligencia emocional $(d=1.14)$ y $(d=1.5)$ respectivamente. Los participantes del primer estudio fueron universitarios al igual que en este estudio; no obstante, en el segundo, se trabajó con una población en inicio de la pubertad y adolescencia. Esto demuestra que un programa de IE propicia la posibilidad de una tendencia al cambio en el comportamiento de las personas a pesar de la edad.

Respecto a la importancia y consistencia de un programa de intervención con el número de sesiones adecuadas, se encontró que el programa cumple con este criterio (10 sesiones con 120 
minutos c/u). De la misma manera, Ramos et al. (2009) realizó una intervención con una cantidad de sesiones parecida (10), lográndose resultados positivos. Sin embargo, la investigación de Sigüenza-Marín, Fonseca-Pedrero y Perez-Albeniz (2019) constó de 10 sesiones con un tiempo de duración de 60 minutos, por lo que no tuvo un impacto en su población; esto indicaría que el número de sesiones y el tiempo administrado influye en los resultados de un programa.

Al momento de la aplicación del pretest, el objetivo era identificar las puntuaciones de las dimensiones de IE antes del programa ART-EMO en los voluntarios. Se obtuvo como resultado que, los voluntarios tenían puntuaciones más altas en las dimensiones Interpersonal y Manejo de Estrés. Estos hallazgos son similares a los obtenidos por otros estudios (Gaspar, 2015; Huemura, 2018; Mamani-Benito et al., 2018), cuyas puntuaciones fueron altas en las dimensiones ya mencionadas (Interpersonal: $\mu=85.29$, Manejo de estrés: $\mu=85.71$ ), (Manejo de estrés: $\mu=102.9$ ) y (Interpersonal: $\mu=17.33$, Manejo de estrés: $\mu=16.58$ ) respectivamente. Lo anterior podría explicarse con que, al ser voluntarios, requieren de una IE medianamente desarrollada y de un perfil específico, el cual se caracteriza por una actitud positiva y habilidades como la asertividad, la capacidad para trabajar en equipo, el compromiso, la disposición, la empatía, el liderazgo, la paciencia, la proactividad y la tolerancia (Huerta, 2017; Poyatos, 2013). Entonces, al desarrollar cierto perfil e integrarse a un voluntariado por decisión propia, favorece su bienestar y satisfacción con lo que realizan (Huerta, 2017). Por ende, resulta necesario cuando se trabaja con población infantil, pues los niños están en una edad en la que construyen sus conocimientos, desarrollan su mundo afectivo; por lo que necesitan de un modelo que les enseñe a identificar sus emociones y ser su soporte en situaciones complejas (Poyatos, 2013; Zambrano et al., 2017).

Finalmente, se buscó comparar las puntuaciones de las dimensiones de IE antes y después del programa ART-EMO en los participantes. En primer lugar, el programa generó un impacto grande en la dimensión Intrapersonal ( $d=1.04$ ) (Ventura-León, 2018). Este hallazgo indica que los voluntarios identificaron la importancia de trabajar el aspecto personal, permitiéndoles incrementar su habilidad para entender y expresar sus sentimientos e identificar puntos fuertes y de mejora y, de esta manera, puedan brindar un servicio de calidad a los niños. Esto ratifica los resultados obtenidos por Mamani-Benito et al. (2018) donde se lograron un incremento en esta dimensión y un efecto grande $(d=1.5)$, al igual que en el estudio de Castillo (2019). El autor trabajó con una población femenina, lo cual se asemeja a la cantidad (90\%) de participantes mujeres del presente programa. Mientras que el trabajo de Hernández, Fernández y Fernández (2020), quienes realizaron 
un programa con hombres reclusos de un penal, no obtuvo un efecto significativo. Aquello indicaría que quizás, al ser en su mayoría mujeres, facilitó la reflexión de sus emociones para dialogar durante las actividades. Por otro lado, respecto a la edad, Coz et al. (2020) realizaron una intervención en niños utilizándose estrategias similares a las del presente programa, sin evidenciarse un incremento en dicha dimensión. Esto podría deberse a que los menores poseen una capacidad de abstracción limitada y las actividades no se adecuaron a ellos; a diferencia de los jóvenes, quienes expresan con facilidad sus ideas y emociones.

En segundo lugar, en la dimensión Interpersonal, el tamaño del efecto también fue grande ( $d=.90)$ (Ventura-León, 2018). Este hallazgo indica que los voluntarios incrementaron su capacidad para comprender las emociones de los demás para relacionarse de forma positiva con ellos al trabajar en equipo. El desarrollo de esta área se asemeja con el estudio de Coz et al. (2020) y en el programa de Rojas y Galván (2020), donde el programa influyó positivamente en esta dimensión, en el trabajo con niños y universitarios respectivamente; por esa razón, las actividades fomentaron el trabajo en grupo, desenvolvimiento y socialización. Esto podría demostrar que, en un programa de educación emocional, es importante contar con actividades participativas donde se enfatice la interacción entre pares y no solo la exploración individual, puesto que la relación con los demás facilita el desarrollo de habilidades sociales (Betina \& Norma, 2017; Mesa, 2018) y permite que el sujeto aprenda a comprender las emociones de otros y relacionarse adecuadamente, lo cual brinda beneficios a nivel personal y grupal (Rojas \& Galván, 2020).

Respecto a la dimensión Adaptabilidad, el impacto del programa en los participantes fue grande ( $d=1.01)$ (Ventura-León, 2018). Este hallazgo indica que los voluntarios incrementaron su habilidad para ser flexibles y encontrar soluciones efectivas a situaciones de cambio propias de su labor. Esto se corrobora con la investigación de Gaspar (2015), donde se obtuvo un tamaño del efecto también grande en esta dimensión $(d=1.1)$. Los participantes de dicha intervención fueron estudiantes de la carrera de ingeniería, quienes emplearon computadoras durante las sesiones; mientras que los participantes del presente programa fueron en su mayoría estudiantes de psicología y las dinámicas eran de carácter vivencial, donde se utilizó recursos artísticos. Si bien ambos grupos obtuvieron un incremento en esta dimensión, esto podría deberse a que el conjunto de ingeniería estaba más familiarizado con el uso de la tecnología; lo que facilitó la ejecución de las tareas al emplear herramientas ya conocidas. Lo mismo ocurrió con los de psicología, quienes están más expuestos a desarrollar actividades con material lúdico. 
En la dimensión Manejo de estrés, el impacto del programa fue moderado $(d=.55)$ (VenturaLeón, 2018), lo que indica que los voluntarios potenciaron su forma de reaccionar ante las adversidades del entorno, aunque es necesario que aprendan formas adecuadas de hacer frente a situaciones de tensión. El manejo de estrés demanda varios aspectos de frustración y ansiedad manifestado en cambios fisiológicos, y al desarrollar un programa en corto tiempo, es complejo modificar estos cambios y, más aún, un aspecto de la personalidad. Asimismo, se encontraron diferentes programas donde la dimensión Manejo del estrés no tuvo efectos, tales como el de Huemura (2018) ( $d=.3$ ), cuya dificultad de modificación se subyace en un estrés acentuado de los participantes; o actividades relativamente nuevas para los participantes que generaban estrés en vez de disminuirlo (Coz et al., 2020).

Por último, en la dimensión Estado de ánimo general, el efecto fue moderado $(d=.65)$ (Ventura-León, 2018). Este hallazgo indica que los voluntarios lograron disfrutar de las actividades y mantener un estado de ánimo positivo ante las circunstancias. Estos resultados se asemejan al estudio sobre inteligencia emocional y técnicas artísticas de Pazo (2018), lo que sugeriría que estas técnicas permiten desarrollar habilidades ligadas a la IE.

Dentro de las implicancias del estudio a partir de los resultados encontrados, la institución puede generar planes de mejora como capacitaciones, campañas y talleres que promuevan el desarrollo de las emociones en las personas que brindan servicios; porque se ha demostrado que están altamente implicadas con el aumento de la producción en una empresa, en este caso una institución que cuenta con voluntarios para trabajar con niños (Villegas, Neme \& García, 2020; PMK Digital Learning, 2018). Por otro lado, se sabe que la sensibilización y conciencia de temas emocionales contribuye al clima de trabajo en una institución y, más aún, cuando el grupo en estudio son personas que brindan apoyo a otras. Por ello, el desarrollo de sus emociones es importante para su autoconocimiento y trato con los demás, encontrándose que la modificación del aspecto Intrapersonal mejora el bienestar personal y, el de Adaptabilidad, su flexibilidad ante situaciones de cambio.

Si bien el estudio contó con hallazgos relevantes, existen algunas limitaciones a considerar, como el contar con un solo grupo, por lo que se recomienda que para una futura replicancia se cuente con un grupo control, con el fin de contrastar sus diferencias y sumar evidencia experimental al estudio de la IE (Chavez \& Esparza-Del Villar, 2020). Otra limitación fue el número de participantes, puesto que para este programa se contó con 10 personas y según los resultados 
obtenidos con un promedio de tres participantes más, el efecto hubiera aumentado. En base a lo mencionado, para evidenciar la estabilidad del constructo se podría aumentar la cantidad de participantes. Por otro lado, el programa fue creado por las investigadoras en base a referentes del tema y conocimiento previo, además de atravesar por una revisión de cinco jueces para hacerlo válido. No obstante, la cantidad de jueces pudo haber sido mayor, pues como mencionan Hyrkäs et al. (2003) y Salinas et al. (2019) un aproximado de 10 a 15 expertos brindarían una mejor valoración del programa de intervención. Por lo tanto, se recomienda que, en futuras investigaciones preexperimentales, los programas pasen por una revisión de expertos teniéndose en cuenta la cantidad mencionada. Por último, si bien las condiciones de aplicación fueron las mismas para cada una de las sesiones, se presentaron variables extrañas (ruidos externos) que interferían con las actividades del programa, las cuales pueden haber afectado las puntuaciones de los participantes (Campbell \& Stanley, 1966). Por este motivo, se recomienda tener medidas preventivas para estas variables externas.

\section{Conclusiones}

Se concluye que el programa ART-EMO fue eficaz debido a que potencializó los niveles de inteligencia emocional en los participantes. Asimismo, se identificó que los voluntarios, antes del programa, tenían puntuaciones más altas en las dimensiones Interpersonal y Manejo de Estrés. De la misma manera, se evidenció que hubo una notable mejora en el área Intrapersonal, lo que demuestra que los voluntarios fortalecieron su habilidad para comprender y expresar sus emociones; no obstante, el impacto fue moderado en Manejo de Estrés, por lo que aún es necesario trabajar en sus recursos personales para hacer frente a situaciones estresantes.

\section{Referencias}

Alarcón, R. (2008). Métodos y diseños de investigación del comportamiento (2da ed.) Lima: Universidad Ricardo Palma.

Ato, M., López, J., \& Benavente, A. (2013). Un sistema de clasificación de los diseños de investigación en psicología. Anales de Psicología, 29 (3), 1038-1059. http://dx.doi.org/10.6018/analesps.29.3.178511

Bar-On, R. (2006). The Bar-On model of emotional-social intelligence (ESI). Psicothema, 18 (1), 13-25. http://www.psicothema.com/psicothema.asp?id=3271 
Betina, A. \& Norma, E. (2017). Relaciones interpersonales positivas: los adolescentes como protagonistas. Psicodebate, 16 (2),73-94. doi: https://doi.org/10.18682/pd.v16i2.598

Campbell, D., \& Stanley, J. (1973). Diseños experimentales y cuasi experimentales en la investigación social. Buenos Aires: Amorrortu Editores.

Castillo, M. (2019). "Meditarte" diseño de un programa de arteterapia y mindfulness para mujeres con dolor crónico en atención primaria [Tesis de Maestría, Universidad Finis Terrae].

https://repositorio.uft.cl/xmlui/bitstream/handle/20.500.12254/1699/Castillo_Magdalena\% 202019.pdf?sequence $=1 \&$ is Allowed $=y$

Chávez, S. \& Esparza-Del Villar, O. (2020). Diseños pre experimentales y cuasi experimentales aplicados a las ciencias sociales y la educación. Enseñanzas e Investigación en Psicología, 2 (2), 167 - 178. https://revistacneip.org/index.php/cneip/article/view/104

Cohen, J. (1988). Statistical power análisis for the behavioral sciencies (2da ed.). Hillsdale, NJ: Erlbaum. https://doi.org/10.4324/9780203771587

Coz, R., Centeno, L., Kasely, Y., \& Rodriguez, F. (2020). Programa de control de impulsos para mejorar la inteligencia emocional en niños. Revista de Asociación Latinoamericana para la Formación y Enseñanza de la Psicología. 8 (24). http://www.integracionacademica.org/37-volumen-8-numero-24-2020/291-programa-de-control-de-impulsospara-mejorar-la-inteligencia-emocional-en-ninos

Domínguez-Lara, S., Merino-Soto, C., \& Gutiérrez-Torres, A. (2017). Estudio estructural de una medida breve de inteligencia emocional en adultos: el EQ-i-M20. Revista Iberoamericana de Diagnóstico y Evaluación-e Avaliação Psicológica, 49 (4), 5-21. https://doi.org/10.21865/RIDEP49.4.01

Gaspar, C. (2015). Efectos del programa PRM en la inteligencia emocional en un grupo de estudiantes dentro del marco prospectivo de universidad saludable [Tesis de Maestría, Universidad Nacional Mayor de San Marcos]. https://cybertesis.unmsm.edu.pe/handle/20.500.12672/4798

Guerrón, E. (2015). Guía didáctica de estrategias en arte terapia para el trabajo con adolescentes entre las edades de 16-18 años que presentan ansiedad del colegio fiscal "Octavio Cordero Palacios” [Tesis de Licenciatura, Universidad del Azuay]. http://dspace.uazuay.edu.ec/bitstream/datos/4969/1/11408.pdf 
Hernández, M., Fernández, C., \& Fernández, C. (21 de abril de 2020). La influencia de la arteterapia sobre la inteligencia emocional en prisión. La mediación artística. https://mediacionartistica.org/2020/04/21/la-influencia-de-la-arteterapia-sobre-lainteligencia-emocional-en-prision/

Hyrkäs, K., Appelqvist-Schmidlechner, K., \& Oksa, L. (2003). Validating an instrument for clinical supervision using an expert panel. International Journal of nursing studies, 40 (6), 619 -625. https://doi.org/10.1016/s0020-7489(03)00036-1

Huemura, S. (2018). Programa para mejorar la inteligencia emocional y correlacionarla con el rendimiento académico en estudiantes de primero de secundaria. Revista Ciencia y Tecnología, 14 $(2)$, 101-113. https://revistas.unitru.edu.pe/index.php/PGM/article/view/2043

Huerta, M. (2017). Proa: hacer voluntariado nunca ha sido tan fácil. [Entrevista a Margarita Briceño]. Canal Ipe. https://www.canalipe.tv/noticias/otros/proa-hacer-voluntariadonunca-ha-sido-tan-facil.

International Labour Organization (ILOSTAT). (2020). https://ilostat.ilo.org/es/interest-involunteering-reached-new-heights-in-march-and-april-2020/

Jiménez, M., Izal, M., \& Montorio, I. (2016). Programa para la mejora del bienestar de las personas mayores. Estudio piloto basado en la psicología positiva. Suma Psicológica, 23 (1), 51-59. https://doi.org/10.1016/j.sumpsi.2016.03.001.

Klein, J-P. (2006). Arte-terapia: creación como proceso de transformación. Papeles de Arteterapia y Educación Artística para la Inclusión Social, (1), 11-18. https://doi.org/10.5209/rev_ARTE.2006.v1.9866

Malchiodi. C. (11 de febrero de 2010). The ten coolest art therapy interventions. Psychology Today. https://www.psychologytoday.com/intl/blog/arts-and-health/201002/the-ten-coolest-arttherapy-interventions

Mamani-Benito, O., Brousett-Minaya, M., Ccori-Zuñiga, D., \& Villasante-Idme, K. (2018). La inteligencia emocional como factor protector en adolescentes con ideación suicida. Duazary, 15 (1). http://dx.doi.org/10.21676/2389783X.2142

Mayo, E. (2018). Vulnerabilidad, ruptura social y arteterapia. Arteterapia. Papeles de arteterapia y educación para inclusión social, 13, 103-117. https://doi.org/10.5209/ARTE.59779 
Mesa, F. (2018). Manual del voluntario. Boys \& Girls Clubs of Puerto Rico. http://bgcpr.org/wpcontent/uploads/2017/06/Manual-del-Voluntarioa-BGCPR-2018.pdf

Murrieta, P., Ruvalcaba, N., Caballo, V., \& Lorenzo, M. (2014). Cambios en la percepción de la violencia y el comportamiento agresivo entre niños a partir de un programa de habilidades $\begin{array}{lllll}\text { socioemocionales. } & \text { Psicología } & \text { Conductual, } & 22 & \text { (3), }\end{array}$ https://www.behavioralpsycho.com/wp-content/uploads/2019/08/10.Murrieta_22-3oa1.pdf

Pazo, I. (2018). La Educación Emocional a través del Arte para menores en acogimiento residencial. Revista de Educación Social, 27. https://eduso.net/res/wpcontent/uploads/2020/06/eduarte_res_27.pdf

Pérez-Fuentes, M., Molero, M., Gázquez, J., \& Mercader, I. (2014). Brief Emotional Intelligence Inventory for Senior Citizens (Eq-i-M20). Psicothema, 26 (4), 524-530. http://dx.doi.org/10.7334/psicothema2014.166

Pérez-Fuentes, M., Molero, M., Gázquez, J., \& Soler, F. (2014). Estimulación de la Inteligencia Emocional en mayores: El Programa PECI-PM. European Journal of Investigation in Health, Psychology and Education, 4 (3), 329-339. http://dx.doi.org/10.1989/ejihpe.v4i3.80

PMK Digital Learning. (10 de noviembre de 2020). El papel de la inteligencia emocional en los negocios de hoy. PMK Digital Learning. https://pmkvirtual.com/blog/inteligenciaemocional/

Poyatos, J. (2013). El desarrollo de habilidades y competencias profesionales en voluntariado internacional. Voluntariado Internacional Eu Aid Volunteers. https://www.voluntare.org/

Quintero, L., Galindo, G., Bonilla, J., \& Rabago, B. (2020). Relación entre funciones ejecutivas y la conducta impulsiva en adolescentes: Estudio comparativo. Salud Ment, 43 (4), 175-180. https://doi.org/10.17711/sm.0185-3325.2020.024

Quiroga, C., \& Chamblás, I. (2014). Inteligencia emocional en jóvenes voluntarios y no voluntarios del Gran Concepción. Perspectivas, 167-183. http://dx.doi.org/10.29344/07171714.25.423

Ramos, N., Hernandez, S., \& Blanca, M. (2009). Efectos de un programa integrado de Mindfulness e Inteligencia emocional sobre las estrategias cognitivas de regulación emocional. Ansiedad y estrés, 15 (2-3), 207-216. https://dialnet.unirioja.es/servlet/articulo?codigo=3095135 
Reyes Bossio, M., Raimundi, M. J., \& Gómez Correa, L. (2012). Programa de entrenamiento en habilidades psicológicas en jugadoras de voleibol de alto rendimiento. Cuadernos de Psicología del Deporte, 12 (1), 9-16. https://revistas.um.es/cpd/article/view/162581

Rojas, G., \& Galván, L. (2020). Arteterapia: una experiencia de implementación remedial voluntaria en universitarios. Revista Iberoamericana para la Investigación y el Desarrollo Educativo, 21 (11). https://doi.org/10.23913/ride.v11i21.687

Ros-Morente, A., Alsinet, C., Torrelles, C., Blasco-Belled, A., \& Jornada, N. (2018). An examination of the relationship between Emotional Intelligence, Positive Affect and Character Strengths and Virtues. Anales de psicología, 34 (1), 63-67. http://dx.doi.org/10.6018/analesps.34.1.262891

Salinas, K., Valencia, A., García, R., \& Gil, F. (2019). Propuesta de validación por jueces expertos de una intervención cognitivo-conductual para cuidadores primarios de pacientes con $\begin{array}{lllll}\text { cáncer. } & \text { Salud } & y & \text { educación, } & 15\end{array}$ (8), https://repository.uaeh.edu.mx/revistas/index.php/ICSA/article/download/4914/6902/

Sánchez-Gómez, M., Roures, A., Flor-Arasil, P., \& Bresó, E. (2021). Propuesta de intervención para el desarrollo de la inteligencia emocional en menores en situación de riesgo y vulnerabilidad social. Apuntes Universitarios, $11 \quad$ (2), $194 \quad$ - 213. https://doi.org/10.17162/au.v11i2.648

Sigüenza-Marín, V., Fonseca-Pedrero, E., \& Perez-Albeniz, A. (2019). Implementación y evaluación de un programa de inteligencia emocional en adolescentes. Universitas Pshychologica, 18 (3). https://doi.org/10.11144/Javeriana.upsy18-3.iepi

Trujillo-Torrealva, D., \& Reyes-Bossio, M. (2019). Programa basado en mindfulness para la reducción de la ansiedad precompetitiva en deportistas de artes marciales. Retos, 36, 418426. http://doi.10.47197/retos.v36i36.66589

Ventura-León, J. (2018). Otras formas de entender la "d" de Cohen. Revista Evaluar, 18 (3), 7378. https://doi.org/10.35670/1667-4545.v18.n3.22305

Villegas, D., Neme, S., García, A. (2020). La Influencia de las Emociones y el Impacto en la Productividad de los Trabajadores de una Microempresa Comercializadora de Plásticos. European Scientific Journal, 16 (10), 56-69. https://doi.org/10.19044/esj.2020.v16n10p56 
Zambrano, D., Chinchay, P., Arce, C., \& Sánchez, E. (2017). Crea + Talento creando cambio no solo en los niños, sino también en los voluntarios: El fomento de las competencias laborales de los voluntarios dentro de la organización. http://hdl.handle.net/20.500.12404/15672 